localisation. However, selective sampling is invasive and technical problems may be encountered. The attempt to show a GHRH concentration gradient across the tumour bed at surgery was only partly successful, probably as a result of the considerable tissue manipulation required to gain access to the feeding and draining vessels. Scanning with labelled octreotide is a useful means of identifying neuroendocrine and gastrointestinal endocrine tumours and can support the notion that an identified mass is the source of GHRH. ${ }^{3}$

Of the 39 fully documented cases of acromegaly due to extracranial tumours synthesizing GHRH identified by Faglia, ${ }^{1} 21$ were bronchial carcinoids, nine were islet cell tumours of the pancreas and three were duodenal or jejeunal carcinoids; 17 had metastases. These tumours were mostly large.

1 Faglia G, Arosio M, Bazzoni N. Ectopic acromegaly. Endocrinol Metab Clin North Am 1992; 21: 575-95.

2 Sano T, Asa SL, Kovacs K. Growth hormone-releasing hormone-producing tumors: clinical, biochemical, and morphological manifestations. Endocrine Rev 1988; 9; $357-73$.

3 Lamberts SWJ, Bakker WH, Reubi JC, Krenning EP. Somatostatin-receptor imaging in the localization of endocrine tumors. $N$ Engl f Med 1990; 323: 1246 -9.
Where full excision is not possible octreotide has proved effective in producing sustained improvement in symptoms and biochemically measured parameters, whereas bromocriptine or pergolide generally have not. ${ }^{1,4}$

\section{Final diagnosis}

Acromegaly secondary to a bronchial carcinoid tumour secreting GHRH.

Keywords: growth hormone, bronchial tumour, acromegaly, octreotide

Our thanks to Professor M Scanlon and Dr M Lewis who performed the GHRH assays, to Dr D Parker who carried out the octreotide scan and $\mathrm{Mr}$ AS Soorae who excised the tumour.

4 Melmed S, Ziel FH, Braunstein GD, Downs T, Frohman LA. Medical management of acromegaly due to ectopic production of growth hormone-releasing hormone by a carcinoid tumour. F Clin Endocrinol Metab 1988; 67: 395-9.

\title{
Acute abdomen after total hip replacement
}

\author{
M Nashi, B Banerjee
}

A 67-year-old woman was admitted for a routine total hip replacement for advanced osteoarthritis. She had a history of gall bladder stones and had undergone a cholecystectomy 30 years earlier. The patient was a non-smoker and there was no history of alcoholic indulgence. The operative procedure was uneventful but on the first postoperative day, the patient developed upper abdominal pain, nausea and vomiting followed by generalised abdominal pain and distension. Abdominal X-rays showed a few distended loops of bowel in the upper abdomen but no other abnormalities. Laboratory investigations prompted an urgent computed tomography (CT) of the abdomen (figure). The patient's condition rapidly deteriorated and she was transferred to the intensive care unit. The patient died due to respiratory and renal failure on the tenth postoperative day.

\footnotetext{
Tameside Trust Hospital, Fountain Street, Ashton under Lyne, OL6 9RW, UK M Nashi

B Banerjee
}

Accepted 29 March 1996

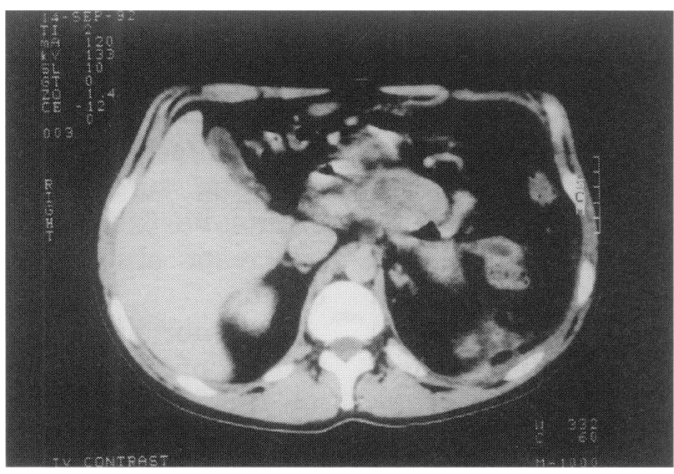

\section{Questions}

1 What does the contrast-enhanced CT scan show?

2 What is the diagnosis?

3 What abnormal laboratory test prompted the CT scan?

4 How is the condition managed?

Figure CT scan of abdomen 
Answers

QUESTION 1

The CT scan through the pancreatic neck and body shows swelling of the gland with focal areas of hypodensity which failed to enhance with contrast agent.

\section{QUESTION 2}

Postoperative acute pancreatitis. Though its incidence is low, the mortality ${ }^{1}$ is over $50 \%$. Most of the reported cases are the result of direct operative trauma to the pancreatic region, but it may also occur after surgical procedures remote from the vicinity of the pancreas, as in our case.

The causes and pathogenesis of postoperative pancreatitis is not well understood. Among the possible causes are hypoxia, hypotension, surgical trauma, and drugs, including anaesthetic agents. ${ }^{2}$

\section{QUESTION 3}

Elevated serum amylase. In our patient serum amylase was raised to $1207 \mathrm{IU} / \mathrm{l}$ (normal value $70-300 \mathrm{IU} / \mathrm{l})$.

\section{QUESTION 4}

The essential treatment lies in early diagnosis and conservative management unless complications amenable to surgical treatment supervene. ${ }^{3}$ It is important that acute pancreatitis should be considered as one of the differential diagnoses in the presence of abdominal pain, vomiting, nausea or intestinal ileus after surgery, including joint replacement.

\section{Discussion}

Acute pancreatitis remains a major cause of morbidity and mortality in the western world. In Britain about $50 \%$ of cases are associated with biliary disease, about $20 \%$ with alcoholism, whilst in the other $20 \%$, the cause is unknown. ${ }^{3}$ Occasionally, hypercalcaemia, hyperlipidaemia and drugs may be associated with an acute attack.

Clinically it presents as acute abdominal pain, usually in the epigastrium or hypochondrium associated with vomiting. It spreads to involve the whole abdomen, which is tender with guarding and rebound tenderness. Severe attacks cause hypotension with sweating and cyanosis. As the condition presents early as an acute abdomen, other causes of acute abdominal pain have to be excluded (box 1).

The main complications of acute pancreatitis are shown in box 2 . Investigations include serum amylase which is elevated on the first day of the disease and thereafter falls rapidly. A value of $1200 \mathrm{IU} / 1$ (normal value $70-300 \mathrm{IU} / \mathrm{l}$ ) or greater is virtually diagnostic. ${ }^{4} \mathrm{~A}$ persistently raised serum amylase suggests the development of pseudocysts or pancreatic abscess. Peritoneal fluid also has a high amylase level. Plain X-rays of the chest and abdomen may show nonspecific changes such as distended loop of bowel or pleural effusion. They also exclude the presence of free gas from a
Causes of acute abdomen associated with raised serum amylase

- perforated peptic ulcer

- small bowel obstruction

- mesenteric infarction

- cholecystitis

- dissection of the aorta

Box 1

\section{Complications of acute pancreatitis}

- respiratory failure

- renal failure

- pseudocyst

- pancreatic abscess

- gastrointestinal haemorrhage

- hypocalcaemia

- diabetes mellitus

- intestinal ileus

Box 2

Adverse prognostic factors (within $48 \mathrm{~h}$ of admission)

The presence of three or more of these criteria predicts a severe attack of pancreatitis ${ }^{5}$ :

- age

- white cell count $>55$ years $>15 \times 10^{9} / 1$

- urea

- glucose

- albumin $>16 \mathrm{mmol} / \mathrm{l}$

- $\mathrm{PaO}_{2}$

- calcium

- alanine aminotransferase $>10 \mathrm{mmol} / \mathrm{l}$

- lactic dehydrogenase

$<32 \mathrm{~g} / 1$

$<8 \mathrm{kPa}$

$<2.0 \mathrm{mmol} / 1$

$>100 \mathrm{IU} / 1$

$>600$ IU/1

Box 3

perforated peptic ulcer. Ultrasound and CT scanning are used to confirm the diagnosis, to assess progress and to detect the development of local complications.

The management of acute pancreatitis should be conservative unless complications amenable to surgical treatment supervene and should be directed towards the treatment of shock and respiratory failure and relief of pain. Surgery is indicated when a condition requiring surgery such as perforated ulcer cannot be excluded. It is also indicated for pancreatic abscess and when there is cholecystitis.

The mortality and prognosis depend on the severity of the attack. Adverse prognostic signs are shown in box 3 . The mortality rate varies between $10-20 \%$. Death rates are not affected by the aetiological cause, except that postoperative pancreatitis is particularly dangerous. Death results either from hypovolaemic shock in the early stage or from multiple organ failure at a later stage. 
1 Rains AJH, Ritchie HD. Bailey E Love's Short practice of surgery, 19th edn. London: HK Lewis \& Co Ltd, 1984; p 904 .

2 Lawrence WW. Current surgical diagnosis and treatment. Los Atlos: Lange Medical Publication, 1985; p 32.

3 Wyatt AP. Diagnosis and management of acute pancreatitis. Ann Coll Surg Engl 1974; 54: 299.
4 Robinson RO, Stott R. Medical emergencies, 5th edn. London: Butterworth, 1987; pp 116-9.

5 Leese T, Shaw D, Holliday M. Prognostic markers in acute pancreatitis: can pancreatic necrosis be predicted? Ann $R$ Coll Surg Engl 1988; 70: 227-32.

\title{
An intra-abdominal tumour
}

\author{
A Rehm, RJ Williams
}

A 51-year-old man presented with a 30-month history of intermittent episodes of breathlessness, dizziness, headaches, palpitations and sweating. In addition, there had been two episodes during which he fainted due to the severity of his symptoms. After the first episode in September 1993 he consulted a general practitioner who diagnosed hypertension and started treatment with lacidipine. Over the following 14 months his symptoms increased in frequency and severity so that he became afraid to leave his house. In November 1994 he consulted a second general practitioner because of the severity of his symptoms and his blood pressure was found to be 180/ $100 \mathrm{mmHg}$. The treatment was changed to nifedipine but the blood pressure remained elevated. The treatment was therefore modified to bendrofluazide, captopril and lacidipine and he was referred to a consultant physician. The clinical findings revealed a heart rate of 80 beats $/ \mathrm{min}$ (sinus rhythm), a supine blood pressure of $170 / 110 \mathrm{mmHg}$ and an erect pressure of 160/ $100 \mathrm{mmHg}$. No other clinical abnormality was noted.

The following investigations were normal: chest X-ray, electrocardiogram, full blood count, urea, electrolytes and liver function tests. An abdominal ultrasound showed a normal left kidney but the right was absent. One out of two 24-hour urine collections showed a total metadrenaline of $7.9 \mu \mathrm{mol} / \mathrm{specimen}$ (normal range: $0-6.0 \mu \mathrm{mol} / \mathrm{specimen}$ ). Three further 24-hour urine collections revealed normal levels of noradrenaline. Serum noradrenaline was normal. An abdominal computed tomography (CT) scan was arranged (figure 1).

\section{Department of Surgery, \\ North Manchester General Hospital, Delaunays Road, Crumpsall, Manchester M8 6RB, UK \\ A Rehm RJ Williams}

\section{Correspondence to} Mr A Rehm, 7 Guildford Grove, London SE10 8JY, UK

Accepted 29 March 1996

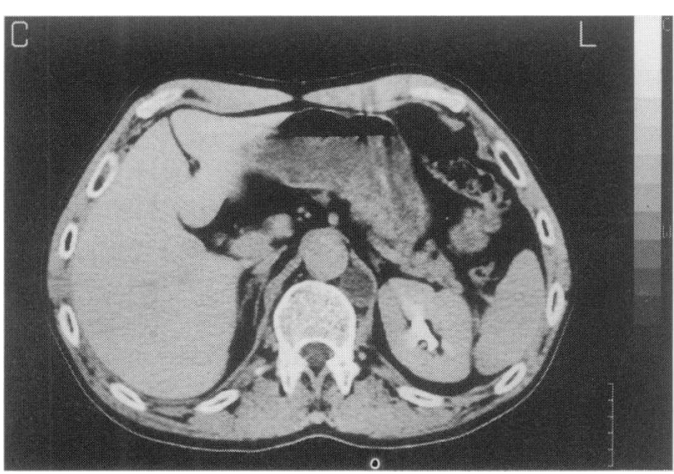

\section{Questions}

1 Which abnormality does the CT scan in figure 1 show?

2 What is the most probable diagnosis considering the case history and clinical findings?

Figure 1 Abdominal CT scan 